\title{
Autoeficacia y felicidad en estudiantes que aspiran a ingresar a la universidad
}

\author{
Self-efficacy and Happiness in Students Wishing \\ to Apply to University
}

Melany Rosario Medina Chugnas

Universidad Peruana de Ciencias Aplicadas, Lima, Perú

(iD https://orcid.org/oooo-0oo2-5489-8966

Andrea Virginia Saxsa Alegre

Universidad Peruana de Ciencias Aplicadas, Lima, Perú

(iD) https://orcid.org/oooo-0oo3-1949-6176

Correspondencia: andrea.vsa13@gmail.com

Miguel Barboza-Palomino

Universidad Privada del Norte, Lima, Perú

(iD https://orcid.org/oooo-ooo1-8045-5491

\section{Resumen}

El estudio buscó determinar la relación entre la autoeficacia y la felicidad en estudiantes de academias preuniversitarias de Lima Metropolitana que aspiran a ingresar a la universidad. Participaron 303 estudiantes preuniversitarios, cuyas edades oscilaron entre los 16 a 23 años $(M=18.05$; $D E=1.252)$, quienes resolvieron la Escala de Autoeficacia General y la Escala de Felicidad. Los resultados evidencian una correlación positiva, alta y estadísticamente significativa entre la autoeficacia y las dimensiones sentido positivo de la vida, satisfacción con la vida y alegría de vivir. A su vez, se halló una relación positiva, moderada y estadísticamente significativa entre la autoeficacia y la dimensión realización personal. Por otra parte, no se encontraron diferencias en la autoeficacia y felicidad considerando el sexo, área de la carrera elegida, tipo y ubicación del colegio de procedencia. Con base en los resultados, se resalta la importancia de desarrollar competencias emocionales en los estudiantes preuniversitarios. Palabras clave: Autoeficacia, felicidad, academias preuniversitarias, estudiantes. 


\begin{abstract}
The study goal was to determine the relationship between self-efficacy and happiness in students from pre-university academies in metropolitan Lima. A sample of 303 pre-university students, whose ages ranged from 16 to 23 years ( $M$ = 18.05; SD =1,252), answered General Self-efficacy Scale and Happiness Scale. The results indicated a positive, high and statistically significant correlation between self-efficacy and the dimensions positive meaning of life, satisfaction with life and joy of living. In turn, a positive, moderate and statistically signifcant relationship was found between self-efficacy and the dimension of personal fulfillment. Also, no differences were found in self-efficacy and happiness considering sex, area of the chosen career, type and location of the high school. Based on the results, the importance of developing emotional competencies in pre-university acadamies students is highlighted.
\end{abstract}

Keywords: Self-efficacy, happiness, pre-university academies, students.

\section{Introducción}

El ingreso a la universidad es una meta importante para los estudiantes que finalizan la escuela, puesto que, continuar estudios superiores los aproxima al éxito profesional y personal (Peñafiel et al., 2019). En el Perú, el ingreso a las universidades públicas y las privadas de mayor prestigio está supeditado al logro de una vacante a través de un concurso de examen de admisión, por lo cual, muchos estudiantes que finalizan la escuela y aspiran a ingresar a una universidad, se preparan en las academias pre universitarias, en donde adquieren o refuerzan conocimientos (Atuncar Yrribari et al., 2015).

Según la Superintendencia Nacional de Educación Superior Universitaria (SUNEDU) (2019), en la ciudad de Lima existen 56 universidades, de las cuales, 45 son privadas y 11 públicas. Si bien, se observa un mayor número de instituciones privadas, muchos estudiantes siguen optando por ingresar a las universidades públicas. Al respecto, Ipsos (2018), en una encuesta que se realizó a jóvenes entre los 15 a 18 años, encontró que, un $77 \%$ planea continuar estudios superiores. De este grupo, el 39\% quiere ir a una universidad pública, el 32\% a una universidad privada y el $29 \%$ a un instituto superior. Aquí, se observa una mayor preferencia por la elección de las universidades públicas. Sin embargo, lograr el ingreso a una universidad pública es una tarea complicada, debido a la exigencia de los exámenes de admisión y al número de vacantes que se ofrecen. Así, por ejemplo, esta es una realidad que se observa en la Universidad Nacional Mayor de San Marcos (UNMSM), donde, en el año 2019, se puso a disposición 4768 vacantes para 24944 postulantes, lo cual dejó a 20176 postulantes sin acceso a esta universidad (Ludena, 2019).

Según el Ministerio de Educación del Perú (MINEDU) (2018), en el 2017, se encontró que, solo el 35.6\% de los estudiantes del nivel de educación secundaria 
tenían los conocimientos necesarios para postular a una universidad o seguir estudios técnicos. En consecuencia, más del 50\% requiere de una preparación adicional para enfrentar un examen de admisión. Frente a esto, las academias preuniversitarias se sitúan como una alternativa idónea para esta población estudiantil. De acuerdo con el diario El Comercio (2010), en una entrevista que se realizó a los jóvenes, la mayoría refirió que la educación recibida en el colegio fue buena, pero no suficiente, razón por la que recurren a instituciones que les aseguren una preparación académica para enfrentar el examen de ingreso. Asimismo, un representante de una academia preuniversitaria, mencionó que, una de las claves para ingresar a la universidad es elegir una buena academia de preparación, que permita al estudiante, incrementar sus posibilidades de ingreso en el menor tiempo posible (Gestión, 2017).

En los últimos años, las academias preuniversitarias se han ido posicionando en el Perú. Estos centros, buscan que los estudiantes consoliden lo aprendido a lo largo de su educación escolar y que amplíen sus conocimientos (Pontificia Universidad Católica del Perú [PUCP], 2019). Por lo tanto, su objetivo principal es que, luego de un determinado tiempo, los estudiantes se encuentren preparados académicamente para enfrentar un examen de admisión. Sin embargo, en la búsqueda de una rigurosa preparación académica, descuidan la atención de otras áreas, como es el aspecto emocional. Al respecto, Mello y Hernández (2019) mencionan que, para un buen rendimiento académico de un estudiante se deben considerar variables cognitivas, afectivas, conductuales e institucionales.

En el entorno pre universitario, el estudiante enfrenta una serie de exigencias y estresores que pueden llegar a afectar su desenvolvimiento académico. Por un lado, según Barraza (2006) existen factores externos como la competencia grupal, la sobrecarga de tareas, el ambiente físico desagradable, la falta de incentivos, los conflictos con los asesores o compañeros y las constantes evaluaciones. Por otra parte, Martínez (2014) considera que no se trabajan las experiencias de fracaso en el intento del ingreso a la universidad, las cuales pueden generar una concepción errónea de sí mismo y la devaluación de sus propias capacidades, afectando también su bienestar subjetivo.

Factores tanto internos como externos influyen en el desenvolvimiento académico y en la percepción que el estudiante tiene de sí mismo. Así, Paoloni y Bonetto (2013) consideran que las creencias que uno tenga de sí mismo, particularmente las que refieren a las percepciones de competencia, constituyen un aspecto relevante vinculado con los éxitos y fracasos de los estudiantes en los contextos de aprendizaje. Esto, puede explicar cómo es que dos personas con el mismo grado de habilidad muestran un desempeño diferente, lo que ocurre debido a que el éxito académico demanda procesos reguladores como la autoevaluación, el automonitoreo y el uso de estrategias metacognitivas de aprendizaje, procesos 
que son influidos por un alto grado de creencia en la propia capacidad o autoeficacia (Pajares \& Schunk, 2001).

Se puede definir a la autoeficacia como la percepción o creencia personal ante las propias capacidades para enfrentarse a una situación determinada. Esta creencia influirá en cómo la persona emplea sus recursos para llevar a cabo una acción y finalmente afronta nuevos retos, consiguiendo resultados deseados (Bandura, 1995). Por esto, se sostiene su gran valor predictivo de la conducta humana, debido a que actúa sobre los patrones de pensamientos, emociones, comportamientos y en la motivación que tenga la persona al momento de enfrentar una situación (Bandura, 1995; Blanco, 2010).

La teoría de la autoeficacia de Bandura explica en el ámbito académico cómo los estudiantes con altas expectativas de autoeficacia gozan de una mayor motivación académica, obtienen mejores resultados, autorregulan eficazmente su aprendizaje y muestran una mayor motivación intrínseca al aprender. En consecuencia, la mejora de las expectativas de autoeficacia incrementa la motivación y el rendimiento (González \& Tourón, 1992). Asimismo, González (2005) menciona como desde que el estudiante ingresa a clases comienza a percibir las creencias de autoeficacia, puesto que, evalúa sus expectativas de éxito ante los objetivos propuestos.

En los últimos años, las teorías de la emoción y el bienestar subjetivo susten$\tan$ que los estados afectivos son una función del estado de desarrollo y de la naturaleza de los objetivos personales (Sansinenea et al., 2008). Estos objetivos personales se relacionan con lo que el individuo quiere lograr en un momento determinado. Tomando en cuenta que las emociones influyen sobre el desarrollo de un objetivo, Alarcón (2006) define a la felicidad como un estado afectivo de satisfacción plena que experimenta subjetivamente el individuo en posesión de un bien anhelado, por lo tanto, una persona es feliz al poseer el objeto anhelado y estos bienes que generan felicidad pueden ser de origen variado (materiales, éticos, estético, psicológicos, entre otros).

En el caso de un estudiante preuniversitario, el ingreso a la universidad deseada implicaría el logro de ese objetivo. Para alcanzarlo, necesita de un rendimiento académico suficiente que le permita responder adecuadamente a las demandas de ingreso. Pero, este éxito académico, según Caballero-García y Sánchez Ruiz (2018), también está relacionado con la felicidad, debido a que esta variable funciona como un predictor. Por ende, en esta etapa de preparación se debe proveer al estudiante una serie de beneficios que le permitan sentirse satisfecho con sus capacidades y logros. Asimismo, Mercado (2014) indica que la felicidad se basa en el planteamiento y cumplimiento de metas; además que está relacionado con la satisfacción con la vida y existe una mejora en las expectativas personales. Es importante mencionar que algunos estudios revelan que un incremento de 
la autoeficacia disminuye las conductas antisociales y las alteraciones emocionales, y al mismo tiempo, influye en la mejora de las conductas de cuidado físico (Galleguillos \& Olmedo, 2017). Al respecto, Bandura (2006) recalca que las conductas de autoeficacia autorregulatoria son claves para entender la competencia emocional de las personas y el éxito en las relaciones interpersonales. Por su parte, Caprara y Steca (2005) reconocen que las creencias de la persona sobre su autoeficacia social, favorecen en la conexión social, la amistad, la cooperación y la conducta prosocial. En cuanto a las conductas de cuidado físico, existen autores que han señalado a la autoeficacia como una variable predictora, mencionándola como uno de los elementos para fomentar el estilo de vida saludable (Blanco et al., 2019). Asimismo, para la recuperación de enfermedades o para evitar sus riesgos potenciales; $y$ en el funcionamiento de distintos campos en busca de cambios positivos sobre los estilos de vida como el realizar ejercicio o deporte, bajar de peso, el manejo de enfermedades crónicas, la elección de carrera y el logro de metas académicas y del mundo laboral (Carrasco \& Del-Barrio, 2002).

Según Bandura (1997), las personas consiguen su bienestar como consecuencia del logro de sus objetivos personales, los cuales dependen en gran parte de sus creencias de autoeficacia. Por lo tanto, un funcionamiento mental positivo depende en gran parte de la competencia autopercibida. Así, los procesos cognitivos, afectivos-motivacionales y las habilidades sociales están muy influidos por las creencias de autoeficacia (Pajares, 2002). Por ende, la autoeficacia es una de las características personales que más promueve el bienestar subjetivo y la felicidad (Gómez et al., 2007). De este modo, se identifica la importancia de la autoeficacia en el desempeño de las diferentes actividades que pueda realizar el estudiante en el ámbito académico y también en su bienestar y felicidad.

En la revisión de la literatura, se han identificado investigaciones con ambas variables en población estudiantil. Al respecto, Morillo (2013) investigó la relación entre la autoeficacia y felicidad en estudiantes universitarios. Con base en sus resultados, señala que, existe una relación directa y significativa entre ambas variables $(\mathrm{p}<. \mathrm{ol})$. Asimismo, encontró un alto porcentaje (51.26\%) de estudiantes con un nivel de autoeficacia medio. Por su parte, Rosales (2017), reportó una correlación estadísticamente significativa entre la autoeficacia y felicidad ( $\mathrm{p}<.05)$. De igual forma, indicó diferencias significativas según el género en la dimensión alegría de vivir de la variable felicidad ( $\mathrm{p}<.05$ ), a favor de los hombres.

Por otra parte, se ubican estudios que han considerado a las variables autoeficacia o felicidad de forma independiente. Así, por ejemplo, Ornelas et al. (2013) investigaron la autoeficacia percibida en la conducta académica de estudiantes universitarias de México que cursaban el primer ciclo académico. Los resultados evidencian que los perfiles de autoeficacia percibida son muy similares entre las estudiantes de las diferentes carreras profesionales. Además, 
se observó que los perfiles de autoeficacia actual, deseada y alcanzable están relacionados. Es decir, a mayor autoeficacia percibida existe un mayor deseoy mayores posibilidades de ser eficaz. En la misma línea, Covarrubias-Apablaza et al. (2019) analizaron la relación entre la autorregulación del aprendizaje y autoeficacia general en las dimensiones de las metas académicas de estudiantes universitarios chilenos. Los resultados muestran que las correlaciones fueron positivas y significativas. Asimismo, la sub dimensión metas de aprendizaje, es la que presenta mejores indicadores para explicar las metas académicas, considerando la autorregulación del aprendizaje como influyente, más que la autoeficacia.

Por su parte, Morales y Giménez (2019) evaluaron los niveles de autoeficacia percibida en universitarios españoles tomando en cuenta los cursos académicos (desde el primer ciclo hasta el máster). Entre sus resultados, reportan que existen diferencias de la autoeficacia según sexo, siendo los hombres quienes obtienen un mayor puntaje. En cuanto al curso, no se encontraron diferencias estadísticamente significativas, sin embargo, se identificó una variabilidad descendiente, con ligeros repuntes de niveles de autoeficacia, el cual se incrementa considerablementeal llegar al máster.

Por otro lado, Caycho y Castañeda (2015) analizaron la relación entre la felicidad y el optimismo en adolescentes y jóvenes peruanos y paraguayos. Los resultados de su investigación, arrojaron una relación positiva y significativa del optimismo con las variables de felicidad. No se observaron

diferencias estadísticamente significativas en la felicidad y optimismo de acuerdo con el sexoy la nacionalidad. Además, Alarcón y Caycho (2015) relacionaron la gratitud y felicidad en jóvenes de universidades públicas y privadas de Lima Metropolitana. Los resultados evidencian que los factores de la gratitud, reciprocidad y obligación moral correlacionan positiva y significativamente con la felicidad $(\mathrm{r}=.294, \mathrm{p}<.01$; $\mathrm{r}=.293, \mathrm{p}<.01$ ), así como, con los factores sentido positivo de la vida, satisfacción con la vida y realización personal de la variable felicidad. Por otro lado, no se evidenciaron diferencias estadísticamente significativas en la gratitud y la felicidad entre hombres y mujeres.

Como se ha expuesto, no se ha estudiado la relación entre las variables autoeficaciay felicidad en el contexto de los estudiantes preuniversitarios. En ese sentido, el objetivo del presente estudio es determinar la relación entre la autoeficacia y felicidad en estudiantes de academias preuniversitarias de Lima Metropolitana que aspiran a ingresar a la universidad. Asimismo, como objetivo específico se plantea comparar la autoeficacia y felicidad de acuerdo con el sexo, el área de la carrera elegida, el tipo y la ubicación del colegio de procedencia.

\section{Método}

Se realizó una investigación empírica empleando la estrategia asociativa retrospectiva (Ato et al., 2013).

\section{Participantes \\ Participantes}


Los participantes del estudio fueron 303 estudiantes deacademias preuniversitarias, 151 varones y 152 mujeres, cuyas edades estuvieron comprendidas entre los 16 a 23 años $(\mathrm{M}=18.05$; $\mathrm{DS}=1.252$ ). La muestra se estimó a través del programa G-power (versión 3.1.9.2), con una potencia de .95 $\mathrm{y}$ un margen de error de .05 .
Se utilizó el tipo de muestreo no probabilístico por conveniencia, debido a que, la elección de la muestra se dio a partir de la participación voluntaria de los estudiantes preuniversitarios que cumplían con las características solicitadas (Kerlinger \& Lee, 2002). En la Tabla 1, se presentan los datos sociodemográficos de los participantes.

Tabla 1.

Datos sociodemográficos de los participantes

\begin{tabular}{|lcc|}
\hline \multicolumn{1}{|c|}{ Característica } & F & $\%$ \\
\hline Área de la carrera elegida & & \\
Letras & 116 & $38.3 \%$ \\
Ciencias & 187 & $61.7 \%$ \\
\hline Tipo de colegio de procedencia & & \\
Estatal & 182 & $60.1 \%$ \\
Particular & 121 & $39.9 \%$ \\
\hline Ubicación del colegio de procedencia & & \\
Lima & 277 & $91.4 \%$ \\
Provincia & 26 & $8.6 \%$ \\
\hline
\end{tabular}

\section{Medidas}

Escala de Autoeficacia general de Schwarzer y Jerusalem (1996)

La versión original fue propuesta por Schwarzer y Jerusalem (1996) y adaptada en el Perú por Grimaldo (2005). Está compuesta por 10 ítems, con un formato de respuesta tipo Likert de 4 alternativas ( $1=$ Incorrecto hasta $4=$ Cierto) que se agrupan en un factor. Para fines de este estudio, se realizó un Análisis Factorial Confirmatorio (AFC), a través del cual se verificó la estructura unidimensional de 10 ítems. Asimismo, la consistencia interna de la escala obtuvo un coeficiente de Alfa de Cronbach de $\alpha=.795$, considerándose un valor aceptable (Cicchetti, 1994).
Escala de Felicidad de Lima (EFL)

Fue elaborada por Alarcón en el 2006. Está compuesta por 27 ítems, con un formato de respuesta tipo Likert con cinco alternativas ( $1=$ Totalmente en desacuerdo hasta $5=$ Totalmente de acuerdo) que se agrupan en 4 factores: 1) Sentido positivo de la vida (SPV), 2) Satisfacción con la vida (SV), 3) Realización personal (RP), y, 4) Alegría de vivir (AV). En este estudio, se realizó un AFC que confirmó las 4 dimensiones originales, pero se decidió eliminar el ítem 11, ya que presentó una carga factorial menora .30. Asimismo, se evaluó la consistencia interna, encontrando valores altos entre .80 y .89 . y moderados entre.70 y 79 (Cicchetti, 1994). De esta forma, se obtuvo un valor alto en la dimensión sentido positivo $(\alpha=.874)$ y valores moderados 
en las dimensiones satisfacción con la vida $(\alpha=.786)$, realización personal $(\alpha=$ $.769)$ y alegría de vivir $(\alpha=.732)$.

\section{Procedimiento}

El proyecto fue aprobado por la Universidad Peruana de Ciencias Aplicadas. Para su ejecución, en primer lugar, se contactó y solicitó la autorización a los responsables de tres academias preuniversitarias. Después, se procedió a la planificación de un cronograma de recolección de datos.

La aplicación de las escalas fue realizada en las mismas academias preuniversitarias y estuvo a cargo de los responsables de la investigación, quienes explicaron los objetivos de la investigación, la forma de la devolución de los resultados, la participación voluntaria y entregaron previamente a los participantes un consentimiento informado.

Una vez recolectado los datos, se trasladó la información a una base de datos en Excel para delimitar las características de la población con base en los datos sociodemográficos. Después, se realizaron los análisis descriptivosy de correlación con el apoyo del Statistical Package for the Social Sciences (SPSS v. 25). En el proceso de análisis, se examinó la distribución normal a través de la prueba de KolmogorovSmirnov. Con base en el resultado de este análisis, se decidió utilizar el coeficiente de correlación de Spearman y se interpretó la magnitud de la correlación considerando la propuesta de Hemphill (2003): bajo $(<.20)$, moderado $(.20$ a .30$)$ y alto
(> .30). A su vez, se empleó la prueba no paramétrica de U de Mann Whitney, a fin de comparar la autoeficacia general y la felicidad en función de las variables sociodemográficas. Finalmente, se estimó el coeficiente de probabilidad de superioridad (PS) como una medida del tamaño del efecto (TE) y se interpretó los valores obtenidos considerando la propuesta de Grissom (1994): pequeño (>.56), mediano $(>.64)$ y grande $(>.71)$.

\section{Resultados}

\section{Análisis correlacional}

Se analizó la normalidad de la distribución de los puntajes mediante la prueba de Kolmogorov-Smirnov. De esta forma, se encontró que los p-valor de la variable autoeficacia y las dimensiones de la variable felicidad fueron menores a .05, lo cual señala la ausencia de normalidad. Con base en este resultado, se decidió hacer uso del coeficiente de correlación de Spearman. Este análisis demuestra una correlación positiva, estadísticamente significativa y con un tamaño de efecto alto entre la autoeficacia general y las dimensiones sentido positivo de la vida (SPV), satisfacción con la vida (SV) y alegría de vivir (AV) de la variable felicidad. A su vez, se evidencia una correlación positiva, estadísticamente significativa y con un tamaño de efecto moderado entre la autoeficacia general y la dimensión realización personal (RP) de la variable felicidad (Tabla 2). 
Tabla 2.

Correlaciones entre el cuestionario de Autoeficacia General (AG) y Felicidad de Lima (FL)

\begin{tabular}{|ccccc|}
\hline $\begin{array}{c}\text { Escala } \\
{[\text { IC95\%] }}\end{array}$ & SPV & SV & RP & AV \\
\hline $\begin{array}{c}\text { Autoeficacia } \\
{[31.71-32.69]}\end{array}$ & $.365^{* *}$ & $.303^{* *}$ & $.277^{* *}$ & $.393^{* *}$ \\
{$[38.36-40.03]$} & {$[21.01-21.94]$} & {$[18.47-19.48]$} & {$[15.61-16.24]$} \\
\hline
\end{tabular}

$\left.{ }^{* *}\right)$ La correlación es significativa en el nivel o,o1 (bilateral).

\section{Análisis comparativo}

Para el análisis comparativo de acuerdo con variables sociodemográficas, se aplicó la prueba U de Mann-Whitney. Según el sexo, no se encontraron diferencias estadísticamente significativas en autoeficacia. Por su parte, en la dimensión satisfacción con la vida (SV) de la escala de felicidad, se encontraron diferencias estadísticamente significativas $(\mathrm{p}<.05)$ a favor de los hombres. Sin embargo, el análisis del tamaño del efecto a través de la probabilidad de superioridad (PS) muestra que no existen diferencias (Tabla 3 ).

Tabla 3 .

Comparación por sexo

\begin{tabular}{|cccccc|}
\hline & $\begin{array}{c}\text { Hombres } \\
\text { Rango promedio }\end{array}$ & $\begin{array}{c}\text { Mujeres } \\
\text { Rango promedio }\end{array}$ & U & Sig. & PS \\
\hline Autoeficacia & 156.13 & 147.90 & 10853 & 0.413 & 0.47 \\
SPV & 149.74 & 154.24 & 11135 & 0.654 & 0.49 \\
SV & 165.31 & 138.78 & 9466 & $0.008^{*}$ & 0.41 \\
RP & 160.31 & 143.74 & 10221 & 0.099 & 0.45 \\
AV & 160.33 & 143.73 & 10218.5 & 0.097 & 0.45 \\
\hline
\end{tabular}

U= U de Mann Whitney; sig.= significancia estadística; $\mathrm{PS}$ = probabilidad de superioridad

Respecto a la comparación por área a la que postulan, tanto en la autoeficacia, como en las dimensiones de felicidad, no se encontraron diferencias estadísticamente significativas. El análisis de la probabilidad de superioridad también reveló la ausencia de diferencias (Tabla 4). 
Tabla 4.

Comparación por áreas

\begin{tabular}{|cccccc|}
\hline & $\begin{array}{c}\text { Letras } \\
\text { Rango promedio }\end{array}$ & $\begin{array}{c}\text { Ciencias } \\
\text { Rango promedio }\end{array}$ & U & Sig. & PS \\
\hline Autoeficacia & 152.30 & 151.81 & 10811 & 0.962 & 0.50 \\
SPV & 163.37 & 144.95 & 9527 & 0.075 & 0.44 \\
SV & 151.20 & 152.49 & 10753.5 & 0.900 & 0.50 \\
RP & 147.19 & 154.99 & 10287 & 0.450 & 0.47 \\
AV & 153.15 & 151.29 & 10712.5 & 0.856 & 0.49 \\
\hline
\end{tabular}

U= U de Mann Whitney; sig.= significancia estadística; $\mathrm{PS}$ = probabilidad de superioridad

Finalmente, considerando el tipo y autoeficaciay felicidad. La estimación de ubicación de colegio de procedencia, la probabilidad de superioridad también se encontró que no existen diferen- evidenció la ausencia de diferencias cias estadísticamente significativas en (Tabla 5).

Tabla 5 .

Comparación por tipo y ubicación del colegio de procedencia

\begin{tabular}{|cccccc|}
\hline & $\begin{array}{c}\text { Estatal } \\
\text { Rango promedio }\end{array}$ & $\begin{array}{c}\text { Particular } \\
\text { Rango promedio }\end{array}$ & U & Sig. & PS \\
\hline Autoeficacia & 152.49 & 151.26 & 10921 & 0.904 & 0.50 \\
SPV & 147.25 & 159.14 & 10146.5 & 0.247 & 0.46 \\
SV & 145.13 & 162.33 & 9761.5 & 0.093 & 0.44 \\
RP & 148.44 & 157.36 & 10362.5 & 0.384 & 0.47 \\
AV & 145.88 & 161.21 & 9896.5 & 0.133 & 0.45 \\
\hline Lima & Rango promedio & Rango promedio & U & Sig. & PS \\
\hline Autoeficacia & 152.28 & 149.02 & 3523.5 & 0.856 & 0.49 \\
SPV & 154.41 & 126.37 & 2934.5 & 0.118 & 0.41 \\
SV & 152.18 & 150.08 & 3551 & 0.907 & 0.49 \\
RP & 152.83 & 143.19 & 3372 & 0.591 & 0.47 \\
AV & 152.18 & 150.06 & 3550.5 & 0.905 & 0.49 \\
\hline
\end{tabular}

U= U de Mann Whitney; sig.= significancia estadística, $\mathrm{PS}=$ probabilidad de superioridad 


\section{Discusión}

El objetivo del estudio fue determinar la relación entre la autoeficacia y felicidad en estudiantes de academias preuniversitarias de Lima Metropolitana. Los resultados evidencian que la autoeficacia se relaciona significativamente y con un tamaño del efecto alto y moderado con las dimensiones de la variable felicidad. Por lo tanto, se puede afirmar que, cuanto más autoeficaces se perciben los estudiantes, también, perciben un mayor sentido positivo con la vida, satisfacción con la vida, alegría de vivir y realización personal.

En lo que corresponde a la interpretación de los resultados considerando los estudios previos, se encontró un hallazgo similar a lo reportado por Morillo (2013) y Rosales (2017), en cuanto existe una relación positiva y significativa entre la autoeficacia y felicidad. Esto, se podría explicar considerando el sustento que señala que la felicidad se basa en el planteamiento y cumplimiento de metas (Mercado, 2014). En el caso de los estudiantes preuniversitarios, este bien anhelado estaría determinado por el ingreso a la universidad deseada, para lo cual, el estudiante, deberá poner en marcha una serie de comportamientos influenciados por la motivación, patrones de pensamientos, emociones, los cuales devienen de la autoeficacia (Bandura, 1995; Blanco, 2010).

Por otra parte, se encontraron diferencias estadísticamente significativas según el sexo en la dimensión alegría de vivir, a favor de los hombres. Esto, coincide con lo reportado por Rosales (2017) y se infiere que los hombres podrían estar experimentando positivamente la vida, lo que implica sentirse bien en la vida en general (Alarcón, 2006). Para este punto, es importante tomar en consideración la participación de los factores asociados a las condiciones de vida de hombres y mujeres (Russo \& Green 1993). Puesto que, las desigualdades en el género pueden repercutir de alguna manera en los niveles de felicidad que tengan, brecha que debe ser menor en sociedades con igualdad de oportunidades, en comparación con aquellas en donde las oportunidades son menores para las mujeres (Tech-Romer et al., 2008).

De otro lado, los hallazgos de este estudio complementan lo reportado por Ornelas et al. (2013), cuyos resultados evidencian similitud en los perfiles de autoeficacia entre los estudiantes de las diferentes carreras profesionales. Esto, se podría deber a que desde la elección de la carrera el estudiante percibe la creencia de autoeficacia, a partir de la evaluación del éxito de sus objetivos (González, 2005). De este modo, se podría explicar que el área a la que postulan los estudiantes, no sería un determinante en los niveles de autoeficacia.

Por otra parte, considerando el sexo, se encontraron resultados similares a los expuestos por Morales y Giménez (2019), en cuanto existen diferencias de la autoeficacia a favor de los hombres en comparación con las mujeres. Las diferencias encontradas pueden ser explicadas mediante la Teoría Social Cognitiva 
(Bandura, 1999), la que sostiene que las expectativas de autoeficacia son uno de los principales condicionantes en la toma de decisiones de cada persona, lo que determina diferencias. Estas diferencias surgen como resultado del proceso de socialización que influye en la diferencia de percepción que construyen hombres y mujeres con relación a las tareas, actividades y ocupaciones que son apropiadas para cada género.

A diferencia de lo reportado en los trabajos de Alarcón y Caycho (2015) y de Castañeda y Caycho (2015), quienes no encontraron diferencias estadísticamente significativas en la felicidad según el sexo. En la presente investigación, se evidenció diferencias significativas en la dimensión satisfacción con la vida, a favor de los hombres. Lo que implicaría una mayor satisfacción por lo que se ha logrado o se está cerca de alcanzar, es decir, la obtención de un bien anhelado (Alarcón, 2006). Esta diferencia podría explicarse por la desigualdad de oportunidades, ya que, de cierto modo, las mujeres necesitan cumplir ciertos roles impuestos por la sociedad, lo que podría dificultar la consecución de objetivos personales.

La presente investigación no está exenta de limitaciones. En primer lugar, al emplear un muestreo de tipo no probabilístico, los resultados no pueden ser generalizados ni concluyentes para toda la población de estudiantes preuniversitarios de Lima Metropolitana. Por lo tanto, se sugiere que en futuras investigaciones se empleen muestreos de tipo probabilístico. En segundo lugar, en el estudio participaron estudiantes provenientes de academias preuniversitarias enfocadas en brindar una preparación exclusiva para el ingreso a algunas universidades. Por ello, se sugiere que en próximas investigaciones se incluyan a estudiantes de otras academias preuniversitarias.

Considerando la relación existente entre las variables en estudio, es relevante promover el desarrollo de aspectos emocionales en los estudiantes preuniversitarios. De este modo, el estudiante dispondrá de recursos para enfrentarse a las demandas y exigencias del contexto. Por lo tanto, sería propicio incorporar cursos, programas o actividades extracurriculares donde se fomente y refuerce las competencias emocionales de los estudiantes. A su vez, es necesario impulsar el desarrollo de otras investigaciones que indaguen diferentes variables en la población de los estudiantes preuniversitarios.

\section{Conclusiones}

En conclusión, en la presente investigación se encontró una relación significativa y con tamaño del efecto alto y moderado entre la autoeficacia y las dimensiones de la variable felicidad en estudiantes de academias preuniversitarias de Lima Metropolitana. Por otro lado, considerando las variables sociodemográficas, para la autoeficacia, no se encontraron diferencias en relación con el sexo, área a la que postulan, tipo y ubicación de procedencia del colegio. 
Por su parte, en lo que corresponde a la dimensión satisfacción con la vida de la variable felicidad, se evidenció diferencias estadísticamente significativas a favor de los hombres, pero, el análisis del tamaño del efecto revela que no existen diferencias.

Finalmente, se comprende que la autoeficacia y felicidad son variables relacionadas que influyen sobre el proceso de aprendizaje y el desempeño del estudiante en la obtención de un bien anhelado, en este caso, el ingreso a la universidad.

\section{Financiamiento}

El proyecto fue autofinanciado.

\section{Conflictos de interés}

Los autores declaran que no tienen conflictos de interés. 


\section{Referencias}

Alarcón, R. (2006). Desarrollo de una escala factorial para medir la Felicidad. Revista Interamericana de Psicología, 40(1), 99-106. http://pepsic.bvsalud.org/pdf/rip/ v4on1/v4onia1o.pdf

Alarcón, R., \& Caycho, T. (2015). Relaciones entre gratitud y felicidad en estudiantes universitarios de lima metropolitana. Psychología: Avances de la Disciplina. Avances de la disciplina, 9(1), 59-69. https://doi.org/10.21500/19002386.993

Ato, M., López, J., \& Benavente, A. (2013). Un sistema de clasificación de los diseños de investigación en psicología. Anales de Psicología, 29(3), 1038-1059. http:// www.redalyc.org/articulo.oa?id=16728244043

Atuncar Yrribari, J., Izaguirre Maguiña, R., Torres Calderón, A., León Nuñez, C., \& Guevara Minaya, B. (2015). Sistema blending de formación preuniversitaria. Electrónica, 18(2), 104-109. https://revistasinvestigacion.unmsm.edu.pe/index. php/electron/article/view/15291/13239

Bandura, A. (1995). Exercice of personal and collective efficacy. En A. Bandura (Ed.) Self-efficacy in Changing Societies (pp. 1-45). Cambridge University Press. https:// www.doi.org/10.1017/CBO9780511527692.003

Bandura, A. (1997). Self-Efficacy: The Exercise of Control. W. H. Freeman and Company.

Bandura, A. (2006). Adolescent development from an agentic perspective. En F. Pajares \& T. Urdan (Eds.). Self-efficacy beliefs of adolescents (pp. 1-43). IAP Information Age Publishing.

Barraza, M. (2006). Un modelo conceptual para el estudio del estrés académico. Revista Electrónica de Psicología Iztacala, 9(3), 110-129. http://www.psicologiacientifica.com/estres-academico-modelo-conceptual/

Blanco, A. (2010). Creencias de autoeficacia de estudiantes universitarios: un estudio empírico sobre la especificidad del constructo. RELIEVE, 16(1), 1-28. http://www. uv.es/RELIEVE/v16n1/RELIEVEv16n1_2.htm

Blanco, L., Díaz-Leal, A., Ornelas, M., Mondaca, F., \& Solano, N. (2019). La autoeficacia en el cuidado de la salud en la predicción de la satisfacción con la vida. Revista Iberoamericana de Diagnóstico y Evaluación Psicológica, 52(3), 53-65. https://doi.org/10.21865/RIDEP52.3.05 
Caballero-García, P.A., \& Sánchez-Ruíz, S. (2018). La felicidad en estudiantes universitarios. ¿Existen diferencias según género, edad o elección de estudios? Revista Electrónica Interuniversitaria de Formación del Profesorado, 21(3), 1-18. https:// doi.org/10.6o18/reifop.21.3.336721

Carrasco, O., \& Del-Barrio, V. (2002). Evaluación de la autoeficacia en niños y adolescentes. Psicothema, 14(2), 323-332. http://www.psicothema.com/pdf/727.pdf

Caprara, G., \& Steca, P. (2005). Affective and social self-regulatory efficacy beliefs as determinants of positive thinking and happiness. European Psychologist, 10(4), 275-286. https://doi.org/10.1027/1016-9040.10.4.275

Caycho, T., \& Castañeda, M. (2015). Felicidad y Optimismo en adolescentes y jóvenes peruanos y paraguayos: Un estudio predictivo. Salud $\mathcal{E}$ Sociedad, 6(3), 250-263. https://doi.org/10.22199/So7187475.2015.0003.00005

Cicchetti, D. (1994). Pautas, criterios y reglas generales para evaluar instrumentos de evaluación estandarizados y estandarizados en psicología. Evaluación Psicológica, 6(4), 284-290. http://doi.apa.org/getdoi.cfm?doi=10.1037/1040-3590.6.4.284

Covarrubias-Apablaza, C., Alcosta-Atognoni, H., \& Mendoza-Lira, M. (2019). Relación de Autorregulación del Aprendizaje y Autoeficacia General con las Metas Académicas de Estudiantes Universitarios. Formación Universitaria, 12(6), 103-113. https://dx.doi.org/10.4067/So718-50062019000600103

El Comercio (o7 de enero de 2010). Jóvenes empezaron clases en academias con un solo sueño: ser universitarios. https://archivo.elcomercio.pe/sociedad/lima/ jovenes-empiezan-clases-academias-sola-idea-universitarios-noticia-392681

Galleguillos, P., \& Olmedo, E. (2017). Autoeficacia académica y rendimiento escolar: un estudio metodológico y correlacional en escolares. ReiDoCrea, 6(14), 156-169. https://www.ugr.es/ reidocrea/6-14.pdf

Gestión (12 de julio de 2017). Claves para lograr ingresar a una universidad pública y no fracasar en el intento. https://gestion.pe/tendencias/management-empleo/ claves-lograr-ingresar-universidad-publica-fracasar-139231-noticia/?ref=gesr

Grimaldo, M. (2005). Validez y confiabilidad de la Escala de Autoeficacia General de Baessler y Schwarzer. Cultura, 19, 213-229. http://www.revistacultura.com. pe/revistas/RCU_19_1_propiedades-psicometricas-de-la-escala-de-autoeficacia-general-de-baessler-schwarzer.pdf 
Gómez, V., Villegas de Posada, C., Barrera, F., \& Cruz, J. E. (2007). Factores predictores de bienestar subjetivo en una muestra colombiana. Revista Latinoamericana de Psicología, 39(2), 311-325. https://www.redalyc.org/articulo.oa?id=80539208

González, A. (2005). Motivación académica. Teoría, aplicación y evaluación. Pirámide.

González, M., \& Tourón, J. (1992). Autoconcepto y rendimiento escolar: sus implicaciones en la motivación y en la autorregulación del aprendizaje. EUNSA

Grissom, R. J. (1994). Probability of the superior outcome of one treatment over another. Journal of Applied Psychology, 79(2), 314-316. https://doi. org/10.1037/o021-9010.79.2.314

Hemphill, J. F. (2003). Interpreting the magnitudes of correlation coefficients. American Psychologist, 58(1), 78-79. https://doi.org/10.1037/ooo3-066X.58.1.78

Ipsos. (19 de octubre de 2018). Imagen y percepción de institutos y universidades. https://www.ipsos.com/es-pe/imagen-y-percepcion-de-institutos-y-universidades

Kerlinger, F., \& Lee, H. (2002). Muestreo y aleatoriedad. En Investigación del comportamiento. McGraw-Hill.

Ludena, L. (o8 de marzo de 2019). Admisión San Marcos: Más de 20 mil postulantes rendirán examen este fin de semana. La República. https://larepublica.pe/ sociedad/1426743-admision-san-marcos-2019-20-mil-postulantes-rendiran-examen-semana-aatp/

Martínez, M. (24 de febrero de 2014). No ingresé a la universidad, ¿Todo está perdido? La República. https://larepublica.pe/archivo/775333-no-ingrese-a-la universidad-todo-esta-perdido

Mello, J. D., \& Hernández, A. (2019). Un estudio sobre el rendimiento académico en Matemáticas. Revista Electrónica de Investigación Educativa, 21, e29, 1-10. https://dx.doi.org/10.24320/redie.2019.21.e29.2090

Mercado, M. (2014). Concepto de felicidad en jóvenes. Ajayu Órgano de Difusión Científica del Departamento de Psicología UCBSP, 12(1), 64-78. http://www.scielo.org. bo/scielo.php?script=sci_arttext\&pid=S2077-21612014000100004\&lng=es\&tlng=es.

Ministerio de Educación (2018). Tasa de transición a educación superior (\% de egresados del nivel educativo anterior). http://escale.minedu.gob.pe/ueetendencias2016 
Morales, F., \& Giménez, J. (2019). Relación entre el curso académico y los niveles de autoeficacia general percibida en universitarios. Magister: Revista de Formación del Profesorado e Innovación Educativa, 31(1), 25-30. https://dialnet.unirioja.es/ servlet/articulo?codigo $=7081936$

Morillo, G. (2013). Autoeficacia y felicidad en ingresantes de una universidad peruana de Trujillo. Revista de psicología, 15(1), 22-36. http://revistas.ucv.edu.pe/index. php/R_PSI/article/view/209/118

Ornelas, M., Blanco, H., Gastelúm, G., \& Chávez, A. (2013). Autoeficacia percibida y conducta académica. (Tesis de licenciatura). Universidad Autónoma de Chihuahua, México.

Pajares, F. (2002). Overview of Social Cognitive Theory and Self-Efficacy. Emory University.

Pajares, F., \& Schunk, D. (2001). Self-beliefs and school success: self-efficacy, self-concept, and school achievement. En R. Riding \& S. Rayner (eds.) Self-perception (pp. 239- 266). Ablex Publishing.

Paoloni, P., \& Bonetto, V. (2013). Creencias de autoeficacia y rendimiento académico en estudiantes universitarios. Revista PsicologíaCientífica.com, 15(5). https://www. psicologiacientifica.com/creencias-de-autoeficacia-y-rendimiento-academico/

Peñafiel, I., Flores, J., \& Vaca, S. (2019). Percepción de estudiantes universitarios con respecto a la elección de su carrera profesional - una decisión de gran trascendencia. Revista Atlante: Cuadernos de Educación y Desarrollo. https://www.eumed. net/rev/atlante/2019/o2/estudiantes-carrera-profesional.html

Pontificia Universidad Católica del Perú (2019). Academias preuniversitarias. De la gran transformación a la hoja de ruta. San Miguel, Perú.: PUCP Vicerrectorado Académico. https://vicerrectorado.pucp.edu.pe/academico/noticias/ academias-preuniversitarias-la-gran-transformacion-la-hoja-ruta/

Rosales, A. (2017). Autoeficacia y Felicidad en estudiantes universitarios de la carrera de una universidad privada de Lima Sur. (Tesis licenciatura). Universidad Autónoma del Perú, Lima, Perú.

Russo, N. F., \& Green, B. L. (1993). Women and mental health. En F. L. Denmakr \& M. A. Paludi (Eds.), Psychology of women: A handbook of issues and theories (pp. 379-436). Greenwood. 
Sansinenea, E., Gil de Montes, L., Agirrezabal, A., Larrañaga, M., Ortiz, G., Valencia, J. F., \& Fuster, M. (2008). Autoconcordancia y autoeficacia en los objetivos personales: ¿Cuál es su aportación al bienestar? Anales de Psicología, 24(1), 121-128. https://www.redalyc.org/articulo.oa?id=167/16724115

Superintendencia Nacional de Educación Superior Universitaria (2019). Universidades. https://www.sunedu.gob.pe/lista-universidades/

Recibido: 11 de marzo de 2021

Revisado: 5 de junio de 2021

Aceptado: 10 de julio de 2021 